\title{
The Borel Sum of Divergent Barnes Hypergeometric Series and its Application to a Partial Differential Equation
}

\author{
By
}

Kunio ICHINOBE*

\begin{abstract}
An explicit form and its analytic continuation around the origin of the Borel sum of the Barnes generalized hypergeometric series ${ }_{q} F_{p-1}$ of divergent type $(q>p)$ is obtained. As an application we give an integral representation of the Borel sum of the formal solution to the Cauchy problem of a certain partial differential equation of non-Kowalevski type.
\end{abstract}

\section{$\S 1 . \quad$ Introduction}

In this paper we shall study the Barnes generalized hypergeometric series ${ }_{q} F_{p-1}$ of divergent type $(q>p)$ which is defined by

$$
{ }_{q} F_{p-1}(\boldsymbol{\alpha} ; \boldsymbol{\gamma} ; z):=\sum_{n=0}^{\infty} \frac{(\boldsymbol{\alpha})_{n}}{(\boldsymbol{\gamma})_{n}} \frac{z^{n}}{n !}, \quad z \in \mathbb{C}
$$

where $\boldsymbol{\alpha}=\left(\alpha_{1}, \ldots, \alpha_{q}\right) \in \mathbb{C}^{q}, \boldsymbol{\gamma}=\left(\gamma_{1}, \ldots, \gamma_{p-1}\right) \in \mathbb{C}^{p-1}$ and the following abbreviations are employed

$$
(\boldsymbol{\alpha})_{n}=\prod_{\ell=1}^{q}\left(\alpha_{\ell}\right)_{n}, \quad(\gamma)_{n}=\prod_{m=1}^{p-1}\left(\gamma_{m}\right)_{n}
$$

Communicated by T. Kawai, June 12, 2000

2000 Mathematics Subject Classification(s): primary 34E05, secondary 33C20, 35C15.

* Graduate School of Mathematics, Nagoya University, Nagoya, 464-8602, Japan.

e-mail: m95006@math.nagoya-u.ac.jp 
with

$$
(c)_{n}=\frac{\Gamma(c+n)}{\Gamma(c)}=\left\{\begin{array}{cc}
c(c+1) \cdots(c+n-1), & n \geq 1 \\
1, & n=0
\end{array} \quad(c \in \mathbb{C}) .\right.
$$

Here $\Gamma$ denotes the Gamma function.

The main interest in this paper is in the structure of the Borel sum of this divergent series, and we shall give an explicit form of the Borel sum (Theorem 2.1 in Section 2) and its analytic continuation around the origin (Theorem 2.2 in Section 2). In these theorems, we only study the case where $\alpha_{i}-\alpha_{j} \notin \mathbb{Z}(i \neq j)$ as a typical case. A result without this condition is given in Theorem 2.3 and further remarks will be given in Section 4 .

Next, Theorem 2.2 will be applied to give an integral representation of the Borel sum (Theorem 5.1 in Section 5) of the divergent formal solution to the following Cauchy problem of a partial differential equation of non-Kowalevski type

$$
\left\{\begin{array}{l}
\partial_{t}^{p} u(t, x)=\partial_{x}^{q} u(t, x), \quad q>p \geq 1, \\
u(0, x)=\varphi(x), \quad \partial_{t}^{j} u(0, x)=0(1 \leq j \leq p-1),
\end{array}\right.
$$

where $t, x \in \mathbb{C}$ and $\varphi(x)$ is a holomorphic function in a neighbourhood of the origin which satisfies the conditions stated in Theorem B in Section 5. (These conditions guarantee the Borel summability of the formal solution.) We will see the kernel function of the integral representation in question can be expressed by a linear combination of ${ }_{p} F_{q-1}$. In Section 6 we will show that it is actually represented by special functions for some particular pairs $(p, q)$.

We have to remark that by the following observation we easily see a relationship between the formal solution of the Cauchy problem (1.2) and the Barnes generalized hypergeometric series ${ }_{q} F_{p-1}$ : Let $\hat{u}(t, x)$ be the formal solution of the Cauchy problem (1.2) which is given by

$$
\hat{u}(t, x)=\sum_{n=0}^{\infty} \varphi^{(q n)}(x) \frac{t^{p n}}{(p n) !} .
$$

Then a formal use of Cauchy's integral formula implies

$$
\hat{u}(t, x)=\frac{1}{2 \pi i} \oint_{|\zeta|=r} \frac{\varphi(x+\zeta)}{\zeta} \sum_{n=0}^{\infty} \frac{(q n) !}{(p n) !} \frac{t^{p n}}{\zeta^{q n}} d \zeta .
$$

Now the relation, $(p n) !=p^{p n} \times \prod_{j=1}^{p}(j / p)_{n}$, implies the following formula in the formal sense,

$$
\hat{u}(t, x)=\frac{1}{2 \pi i} \oint_{|\zeta|=r} \frac{\varphi(x+\zeta)}{\zeta}{ }_{q} F_{p-1}(\boldsymbol{\alpha} ; \boldsymbol{\gamma} ; z) d \zeta
$$


where $\boldsymbol{\alpha}=(1 / q, 2 / q, \ldots, q / q), \boldsymbol{\gamma}=(1 / p, 2 / p, \ldots,(p-1) / p)$ and $z=q^{q} t^{p} / p^{p} \zeta^{q}$.

Here we give a brief history of the theory of $k$-summability or the Borel summability. After the concept of $k$-summability for divergent power series was given by Ramis [Ram] in 1980, the theory of multisummability introduced by Ecalle has been developed extensively by many authors, and it was proved by Braaksma that every formal solution of an analytic ordinary differential equation is multisummable (cf. [Bra], see also Balser [Bal 1] for more detailed history and results).

Recently there have been some attempts to apply the theory of $k$-summability or the Borel summability to the study of divergent formal solutions of partial differential equations of non-Kowalevski type. Firstly, Lutz-Miyake-Schäfke [LMS] studied the formal solution of the Cauchy problem of the complex heat equation, and the Borel summability was characterized in terms of an analytic continuation property and a growth condition to the Cauchy data. For more general Cauchy data this result was generalized by Balser [Bal 2]. In BalserMiyake [BM], they proved a sufficient condition for the Borel summability for divergent series obtained through a certain recursion formula. (They applied their results to a formal solution of the Cauchy problem of a certain partial differential equation which generalizes (1.2).) After that, Miyake [Miy] proved that the sufficient condition obtained in $[\mathrm{BM}]$ is also a neccesary condition for the formal solutions of the Cauchy problem (1.2) to be Borel summable (cf. Theorem B). Therefore, Theorem 5.1 in this paper gives an integral representation of the Borel sum under the conditions given in $[\mathrm{Miy}]$ or $[\mathrm{BM}]$. At the end of this introduction, we note that a part of this paper is published in [MI] without detailed proofs.

\section{$\S 2 . \quad$ Statement of Results}

In this section we shall state our main results concerning the Borel sum and its analytic continuation of the Barnes generalized hypergeometric series of divergent type $(q>p)$ which is given in (1.1),

$$
\hat{f}(z)={ }_{q} F_{p-1}(\boldsymbol{\alpha} ; \boldsymbol{\gamma} ; z)={ }_{q} F_{p-1}\left(\begin{array}{l}
\boldsymbol{\alpha} \\
\gamma
\end{array} ;\right) .
$$

In order to make sense of this series we assume $\gamma_{m} \notin \mathbb{Z}_{\leq 0}$ for all $m \in\{1,2, \ldots$, $p-1\}$ and to avoid the trivial case we assume $\alpha_{\ell} \notin \mathbb{Z}_{\leq 0}$ for all $\ell \in\{1,2, \ldots, q\}$ where $\mathbb{Z}_{\leq 0}:=\{0,-1,-2, \ldots\}$.

It is known that $\hat{f}(z)$ is a formal solution of the following differential equa- 
tion,

$$
E_{q, p-1}[\boldsymbol{\alpha} ; \boldsymbol{\gamma}](u):=\left(\theta \prod_{m=1}^{p-1}\left(\theta+\gamma_{m}-1\right)-z \prod_{\ell=1}^{q}\left(\theta+\alpha_{\ell}\right)\right) u(z)=0
$$

where $\theta=z(d / d z)$.

In the case $q \leq p$, the order of this differential equation is $p$. In the case where any two of $\left\{1, \gamma_{1}, \ldots, \gamma_{p-1}\right\}$ do not differ by an integer, the other $(p-1)$ solutions are given by

$$
u_{j}(z)=z^{1-\gamma_{j}} F_{p-1}\left(\begin{array}{c}
1-\gamma_{j}+\boldsymbol{\alpha} \\
2-\gamma_{j}, 1-\gamma_{j}+\widehat{\gamma}_{j}
\end{array} ; z\right), \quad j=1, \ldots, p-1
$$

where $1-\gamma_{j}+\boldsymbol{\alpha}=\left(1-\gamma_{j}+\alpha_{1}, \ldots, 1-\gamma_{j}+\alpha_{q}\right)$ and $\widehat{\gamma_{j}} \in \mathbb{C}^{p-2}$ is obtained by omitting the $j$-th component from $\gamma$.

Now, our interest is in the case $q>p$. In this case, our formal series $\hat{f}(z)$ is divergent. We want to study the Borel summability of this divergent series. Before stating our results we shall prepare some notations and definitions (cf. [Bal 1]).

1. Sector. For $d \in \mathbb{R}, \beta>0$ and $\rho(0<\rho \leq \infty)$, we define a sector $S=S(d, \beta, \rho)$ by

$$
S(d, \beta, \rho):=\left\{z \in \mathbb{C} ;|d-\arg z|<\frac{\beta}{2}, 0<|z|<\rho\right\}
$$

where $d, \beta$ and $\rho$ are called the direction, the opening angle and the radius of $S(d, \beta, \rho)$, respectively.

2. Gevrey formal power series. For $k>0$, we say that $\hat{u}(z)=$ $\sum_{n=0}^{\infty} u_{n} z^{n}$ belongs to $\mathbb{C}[[z]]_{1 / k}$, which is called the formal power series of Gevrey order $1 / k$, if there exist some positive constants $C$ and $K$ such that for any $n$ we have

$$
\left|u_{n}\right| \leq C K^{n} \Gamma\left(1+\frac{n}{k}\right)
$$

Then it is easily seen that $\hat{f}(z) \in \mathbb{C}[[z]]_{q-p}$.

3. Gevrey asymptotic expansion. Let $k>0, \hat{u}(z)=\sum_{n=0}^{\infty} u_{n} z^{n} \in$ $\mathbb{C}[[z]]_{1 / k}$ and $u(z) \in \mathcal{O}(S)$. Here $\mathcal{O}(S)$ denotes the set of holomorphic functions on a sector $S$. Then we say that

$$
u(z) \cong_{k} \hat{u}(z) \text { in } S,
$$


if for any closed subsector $S^{\prime}$ of $S$, there exist some positive constants $C$ and $K$ such that for any $N$ we have

$$
\left|u(z)-\sum_{n=0}^{N-1} u_{n} z^{n}\right| \leq C K^{N}|z|^{N} \Gamma\left(1+\frac{N}{k}\right), \quad z \in S^{\prime} .
$$

4. Borel summability. For $k>0, d \in \mathbb{R}$ and $\hat{u}(z) \in \mathbb{C}[[z]]_{1 / k}$, we say that $\hat{u}(z)$ is $k$-summable in $d$ direction or Borel summable for short if there exist a sector $S=S(d, \beta, \rho)$ with $\beta>\pi / k$ and $u(z) \in \mathcal{O}(S)$ for which $u(z) \cong_{k} \hat{u}(z)$ holds in $S$. In this case such a function $u(z)$ is called the Borel sum of $\hat{u}(z)$.

Remark 1 . Let $\hat{u}(z) \in \mathbb{C}[[z]]_{1 / k}$ be given.

(i) If $\beta \leq \pi / k$, then there are infinitely many $u$ 's satisfying $u(z) \cong_{k} \hat{u}(z)$ in $S(d, \beta, \rho)$ for any $d$ and some $\rho>0$.

(ii) If $\beta>\pi / k$, then the Borel sum $u(z)$ of $\hat{u}(z)$ does not exist in general, but it is unique if it does exist. In this sense the notion of the Borel sum is well defined, and a characterization of $k$-summability for a given divergent series will be given in Lemma 3.1 in Section 3 (cf. [Bal 1]).

Now our first result is stated as follows.

Theorem 2.1 (Borel sum). Assume that $\alpha_{i}-\alpha_{j} \notin \mathbb{Z} \quad(i \neq j)$. Then $\hat{f}(z)$ is $1 /(q-p)$-summable in any direction $d$ such that $d \neq 0(\bmod 2 \pi)$ and its Borel sum $f(z)$ is given by

$$
f(z)=C_{\alpha \gamma} \sum_{j=1}^{q} C_{\alpha \gamma}(j) \times(-z)^{-\alpha_{j}} F_{q-1}\left(\begin{array}{l}
\alpha_{j}, 1+\alpha_{j}-\gamma \\
1+\alpha_{j}-\widehat{\boldsymbol{\alpha}_{j}}
\end{array} ; \frac{(-1)^{p-q}}{z}\right),
$$

where $z \in S(\pi,(q-p+2) \pi, \infty)$ and

$$
C_{\alpha \gamma}=\frac{\Gamma(\gamma)}{\Gamma(\boldsymbol{\alpha})}, \quad C_{\alpha \gamma}(j)=\frac{\Gamma\left(\alpha_{j}\right) \Gamma\left(\widehat{\boldsymbol{\alpha}_{j}}-\alpha_{j}\right)}{\Gamma\left(\gamma-\alpha_{j}\right)} .
$$

Here we use the following abbreviations:

$$
\Gamma(\boldsymbol{\alpha})=\prod_{\ell=1}^{q} \Gamma\left(\alpha_{\ell}\right), \quad \Gamma\left(\widehat{\boldsymbol{\alpha}_{j}}-\alpha_{j}\right)=\prod_{\ell=1, \ell \neq j}^{q} \Gamma\left(\alpha_{\ell}-\alpha_{j}\right) .
$$

We remark that $C_{\alpha \gamma}(j)=0$ if $\gamma_{i}-\alpha_{j} \in \mathbb{Z}_{\leq 0}$ for some $i$.

Next, our result for the analytic continuation of the Borel sum $f$ is stated as follows. 
Theorem 2.2 (Analytic Continuation of Borel sum). Under the same assumptions as in Theorem 2.1, we have

$$
\begin{aligned}
& \frac{1}{2 \pi i}\left\{f(z)-f\left(z e^{2 \pi i}\right)\right\} \\
& =C_{\alpha \gamma} \sum_{j=1}^{q} \frac{C_{\alpha \gamma}(j)}{\Gamma\left(\alpha_{j}\right) \Gamma\left(1-\alpha_{j}\right)} z^{-\alpha_{j}} F_{q-1}\left(\begin{array}{l}
\alpha_{j}, 1+\alpha_{j}-\gamma \\
1+\alpha_{j}-\widehat{\boldsymbol{\alpha}_{j}}
\end{array} ; \frac{(-1)^{p-q}}{z}\right),
\end{aligned}
$$

where $z \in S(0,(q-p) \pi, \infty)$ and $C_{\alpha \gamma}, C_{\alpha \gamma}(j)$ are the same constants as above.

Remark 2. In 1907, Barnes [Bar] (cf. [Mei]) obtained an asymptotic expansion of linear combinations of generalized hypergeometric function as follows, which is an alternative version of Theorem 2.1.

Theorem A (Barnes). Let $q>p$. Then there are $q$ asymptotic expansions of the following form:

$$
\begin{array}{r}
C_{\alpha \gamma} \sum_{j=1}^{q} C_{\alpha \gamma}(j) \times t^{\alpha_{j}-\alpha_{s}} F_{q-1}\left(\begin{array}{c}
\alpha_{j}, 1+\alpha_{j}-\gamma \\
1+\alpha_{j}-\widehat{\boldsymbol{\alpha}_{j}}
\end{array} ;(-1)^{p-q-1} t\right) \\
\sim t^{-\alpha_{s}} F_{p-1}\left(\begin{array}{l}
\boldsymbol{\alpha} \\
\gamma
\end{array} \frac{-1}{t}\right) \text { as } t \rightarrow \infty, \quad t \in S(0,(q-p+2) \pi, \infty),
\end{array}
$$

where $s=1,2, \ldots, q$.

We remark that $q$ functions on the left hand side are fundamental solutions of the following differential equation

$$
\begin{aligned}
E_{p, q-1}\left[\alpha_{s}, 1\right. & \left.+\alpha_{s}-\gamma ; 1+\alpha_{s}-\widehat{\boldsymbol{\alpha}_{s}}\right](u) \\
& =\left\{\prod_{\ell=1}^{q}\left(\theta+\alpha_{s}-\alpha_{\ell}\right)-z\left(\theta+\alpha_{s}\right) \prod_{m=1}^{p-1}\left(\theta+1+\alpha_{s}-\gamma_{m}\right)\right\} u(z)=0,
\end{aligned}
$$

with $z=(-1)^{p-q-1} t$.

Remark 3. In the case where there exists a pair $\left(\alpha_{i}, \alpha_{j}\right)$ such that $\alpha_{i}-$ $\alpha_{j} \in \mathbb{Z}$, we can give the similar results to Theorem 2.1 where the logarithmic terms appear. In fact, we can prove the following theorem (cf. [Erd 2, p.63], [Luk, p.14]).

Theorem 2.3. Let us assume $\alpha_{2}-\alpha_{1}=m \quad(m \geq 0), \alpha_{j}-\alpha_{1} \notin \mathbb{Z}$ $(3 \leq j \leq q)$ and $\gamma_{k}-\alpha_{1} \notin \mathbb{Z} \quad(1 \leq k \leq p-1)$. Then the Borel sum $f(z)$ is given by

$$
\frac{f(z)}{C_{\alpha \gamma}}=C_{\alpha \gamma}(1)(-z)^{-\alpha_{1}} \sum_{k=0}^{m-1} \frac{\left(\alpha_{1}\right)_{k}\left(1+\alpha_{1}-\gamma\right)_{k}}{\left(1+\alpha_{1}-\widehat{\boldsymbol{\alpha}_{1}}\right)_{k}} \frac{1}{k !}\left(\frac{(-1)^{p-q}}{z}\right)^{k}
$$




$$
\begin{aligned}
& +\sum_{j=3}^{q} C_{\alpha \gamma}(j)(-z)^{-\alpha_{j}} F_{q-1}\left(\begin{array}{l}
\alpha_{j}, 1+\alpha_{j}-\gamma \\
1+\alpha_{j}-\widehat{\boldsymbol{\alpha}_{j}}
\end{array} \frac{(-1)^{p-q}}{z}\right) \\
& +\frac{(-1)^{m}}{m !} C_{\alpha \gamma}\left(2 ; \widehat{\alpha_{1}}\right)(-z)^{-\alpha_{2}} \sum_{n=0}^{\infty} \frac{\left(\alpha_{2}\right)_{n}\left(1+\alpha_{2}-\gamma\right)_{n}}{\left(1+\alpha_{2}-\widehat{\boldsymbol{\alpha}_{2}}\right)_{n}} \frac{1}{n !}\left(\frac{(-1)^{p-q}}{z}\right)^{n} \\
& \quad \times\{\log (-z)+\Psi(n+1)+\Psi(n+m+1) \\
& \left.\quad+\sum_{j=3}^{q} \Psi\left(\alpha_{j}-\alpha_{2}-n\right)-\Psi\left(\alpha_{2}+n\right)-\Psi\left(\gamma-\alpha_{2}-n\right)\right\}
\end{aligned}
$$

where $z \in S(\pi,(q-p+2) \pi, \infty)$ and

$$
C_{\alpha \gamma}\left(2 ; \widehat{\alpha_{1}}\right)=\frac{\Gamma\left(\alpha_{2}\right) \prod_{\ell=1, \ell \neq 1,2}^{q} \Gamma\left(\alpha_{\ell}-\alpha_{2}\right)}{\Gamma\left(\gamma-\alpha_{2}\right)} .
$$

Here $\Psi(z)=\Gamma^{\prime}(z) / \Gamma(z)$ denotes the Psi function and we use the following abbreviations

$$
\Psi\left(\gamma-\alpha_{2}-n\right)=\sum_{k=1}^{p-1} \Psi\left(\gamma_{k}-\alpha_{2}-n\right),
$$

and $\sum_{k=0}^{m-1} \cdots=0$ if $m=0$.

\section{§3. $\quad$ Proof of Theorems 2.1 and 2.2}

In order to prove Theorem 2.1, we use the following lemma for the Borel summability.

Lemma 3.1. Let $k>0, d \in \mathbb{R}$ and $\hat{u}(z) \in \mathbb{C}[[z]]_{1 / k}$. Then the following three statements are equivalent:

(i) $\hat{u}(z)$ is k-summable in d direction.

(ii) Let $g(\xi)$ be the formal $k$-Borel transform of $\hat{u}(z)$

$$
g(\xi)=\left(\hat{\mathcal{B}}_{k} \hat{u}\right)(\xi):=\sum_{n=0}^{\infty} \frac{u_{n}}{\Gamma(1+n / k)} \xi^{n},
$$

which is holomorphic in a neighbourhood of $\xi=0$. Then $g(\xi)$ can be continued analytically in $S(d, \varepsilon, \infty)$ for some positive constant $\varepsilon$ and satisfies a growth condition of exponential order at most $k$ there, that is, there exist some positive number $C$ and $\delta$ such that we have

$$
|g(\xi)| \leq C \exp \left\{\delta|\xi|^{k}\right\}, \xi \in S(d, \varepsilon, \infty) .
$$


(iii) Let $j \geq 2$ and $k_{1}>0, \ldots, k_{j}>0$ satisfy $1 / k=1 / k_{1}+\cdots+1 / k_{j}$. Let $h(\xi)$ be the following iterated formal Borel transforms of $\hat{u}(z)$

$$
h(\xi)=\left(\hat{\mathcal{B}}_{k_{1}} \circ \cdots \circ \hat{\mathcal{B}}_{k_{j}} \hat{u}\right)(\xi) .
$$

Then $h(\xi)$ holds the same properties as $g(\xi)$ above.

In the case (ii), the Borel sum $u(z)$ is obtained after an analytic continuation of the following Laplace integral

$$
u(z)=\left(\mathcal{L}_{k} g\right)(z):=\frac{1}{z^{k}} \int_{0}^{\infty(d)} e^{-(\xi / z)^{k}} g(\xi) d\left(\xi^{k}\right),
$$

where $z \in S(d, \beta, \rho)$ with $\beta<\pi / k$ and $\rho>0$ and the path of integration is taken from 0 to $\infty$ along the half line of argument $d$.

In the case (iii), the Borel sum $u(z)$ is obtained after an analytic continuation of the following iterated Laplace integrals

$$
u(z)=\left(\mathcal{L}_{k_{j}} \circ \cdots \circ \mathcal{L}_{k_{1}} h\right)(z) .
$$

The equivalence of (i) and (ii) was given in [Bal 1] and the equivalence of (iii) with others was proved in [Miy].

First proof of Theorem 2.1. We employ the statement (iii) in Lemma 3.1. Let $h(\xi)$ be the $(q-p)$ times iterated formal 1-Borel transforms of our formal series $\hat{f}(z)$

$$
h(\xi)=\left(\left(\hat{\mathcal{B}}_{1}\right)^{q-p} \hat{f}\right)(\xi)=\sum_{n=0}^{\infty} \frac{(\boldsymbol{\alpha})_{n}}{(\gamma)_{n}(n !)^{q-p}} \frac{\xi^{n}}{n !}={ }_{q} F_{q-1}\left(\begin{array}{c}
\boldsymbol{\alpha} \\
\gamma, 1, \ldots, 1
\end{array} ; \xi\right)
$$

This series is convergent in $|\xi|<1$. Then we can see that $h(\xi) \in \mathcal{O}(\mathbb{C} \backslash[1, \infty))$ and $h(\xi)$ has at most polynomial growth as $\xi \rightarrow \infty$, because $h(\xi)$ satisfies

$$
E_{q,(q-1)}[\boldsymbol{\alpha} ; \boldsymbol{\gamma}, 1, \ldots, 1](h)=0,
$$

which is a Fuchsian equation with singular points $\{0,1, \infty\}$. Therefore $\hat{f}(z)$ is $1 /(q-p)$-summable in any direction $d$ such that $d \neq 0(\bmod 2 \pi)$ and the Borel sum $f(z)$ is given by the following iterated Laplace integrals

$$
\begin{aligned}
f(z)= & \left(\left(\mathcal{L}_{1}\right)^{q-p} h\right)(z) \\
= & \frac{1}{z} \int_{0}^{\infty(d)} \exp \left(-\frac{s_{1}}{z}\right) d s_{1} \frac{1}{s_{1}} \int_{0}^{\infty(d)} \exp \left(-\frac{s_{2}}{s_{1}}\right) d s_{2} \times \cdots \\
& \cdots \times \frac{1}{s_{q-p-1}} \int_{0}^{\infty(d)} \exp \left(-\frac{\xi}{s_{q-p-1}}\right) h(\xi) d \xi
\end{aligned}
$$


where $|d-\arg z|<\pi / 2$ and $\arg s_{j}=\arg \xi=d \neq 0(1 \leq j \leq q-p-1)$. We can regard that $f(z)$ is analytic in $S(\pi,(q-p+2) \pi, \infty)$ by taking analytic continuation of $f(z)$ by rotating the arguments of the paths of these iterated integrals. By a change of variables, we have

$$
f(z)=\int_{0}^{\infty(a)} e^{-u_{1}} d u_{1} \int_{0}^{\infty(0)} e^{-u_{2}} d u_{2} \cdots \int_{0}^{\infty(0)} e^{-u_{q-p}} h(\mathbf{u} z) d u_{q-p}
$$

where $a=d-\arg z$ and $\mathbf{u}=u_{1} \cdots u_{q-p}$.

To calculate this iterated integrals, we employ the Barnes integral representation of $h(\xi)$ (cf. [IKSY]), which is given by

$$
h(\xi)=\frac{C_{\alpha \gamma}}{2 \pi i} \int_{I} \frac{\Gamma(\boldsymbol{\alpha}+\zeta) \Gamma(-\zeta)}{\Gamma(\gamma+\zeta)\{\Gamma(1+\zeta)\}^{q-p}}(-\xi)^{\zeta} d \zeta, \quad|\arg (-\xi)|<\pi,
$$

where $C_{\alpha \gamma}$ is the same constant as in Theorem 2.1. Here the path of integration $I$ runs from $-i \infty$ to $+i \infty$ in such a manner that the poles of $\Gamma(\boldsymbol{\alpha}+\zeta)$ which are ploted by $\bullet$ are on the left side of $I$ and the poles of $\Gamma(-\zeta)$ which are ploted by $\circ$ are on the right side of $I$ (see the figure below).

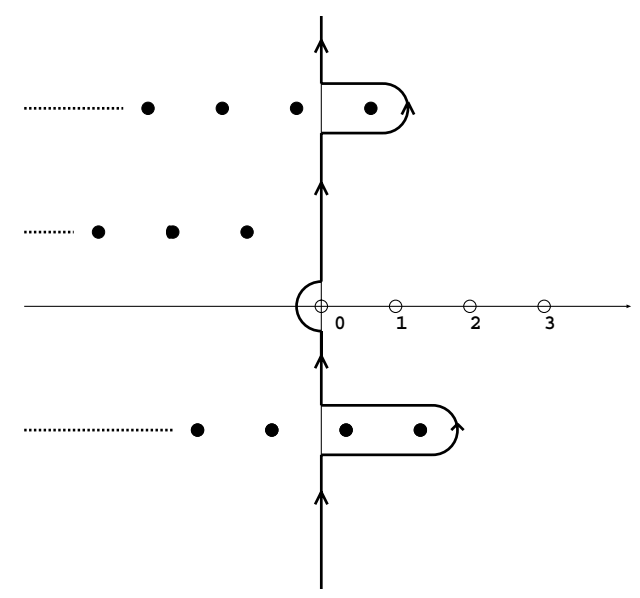

Here we remark that from the expression (3.10) $h(\xi)$ becomes either the sum of the residues at $\{n ; n \geq 0\}$ located on the right side of $I$ or the sum of the residues at $\left\{-\alpha_{j}-n ; n \geq 0,1 \leq j \leq q\right\}$ located on the left side of $I$; thus we obtain a connection formula between a solution at $\xi=0$ and solutions at $\xi=\infty$ of the equation (3.7) (cf. [IKSY]). Moreover by taking residues on the left side of $I$, we can exchange the order of integrations in (3.9), and we obtain the following fundamental formula for the Borel sum

$$
f(z)=\frac{C_{\alpha \gamma}}{2 \pi i} \int_{I} \frac{\Gamma(\boldsymbol{\alpha}+\zeta) \Gamma(-\zeta)}{\Gamma(\boldsymbol{\gamma}+\zeta)}(-z)^{\zeta} d \zeta, \quad z \in S(\pi,(q-p+2) \pi, \infty)
$$


Now, by the residue theorem, we obtain the conclusion as follows. First, by taking residues on the left side of $I$, we have

$$
f(z)=C_{\alpha \gamma} \sum_{j=1}^{q} \sum_{n=0}^{\infty} \frac{\Gamma\left(\widehat{\boldsymbol{\alpha}_{j}}-\alpha_{j}-n\right) \Gamma\left(\alpha_{j}+n\right)}{\Gamma\left(\gamma-\alpha_{j}-n\right)} \frac{(-1)^{n}}{n !}(-z)^{-\alpha_{j}-n} .
$$

Here we notice the formula

$$
\frac{1}{\Gamma(c-n)}=\frac{(1-c)_{n}(-1)^{n}}{\Gamma(c)}, \quad c \in \mathbb{C},
$$

which is obtained from the reciprocal formula

$$
\sin (\pi \zeta)=\pi /\{\Gamma(\zeta) \Gamma(1-\zeta)\}
$$

(cf. [Erd 2, p.3]). Then by using this formula (3.13) to the terms $\Gamma\left(\widehat{\boldsymbol{\alpha}_{j}}-\alpha_{j}-n\right)$ and $\Gamma\left(\gamma-\alpha_{j}-n\right)$, we get

$$
\begin{aligned}
f(z) & =C_{\alpha \gamma} \sum_{j=1}^{q} C_{\alpha \gamma}(j)(-z)^{-\alpha_{j}} \sum_{n=0}^{\infty} \frac{\left(\alpha_{j}\right)_{n}\left(1+\alpha_{j}-\gamma\right)_{n}}{\left(1+\alpha_{j}-\widehat{\boldsymbol{\alpha}_{j}}\right)_{n}} \frac{(-1)^{(p-q) n}}{n !} z^{-n} \\
& =C_{\alpha \gamma} \sum_{j=1}^{p} C_{\alpha \gamma}(j)(-z)^{-\alpha_{j}} F_{p-1}\left(\begin{array}{l}
\alpha_{j}, 1+\alpha_{j}-\gamma \\
1+\alpha_{j}-\widehat{\boldsymbol{\alpha}_{j}}
\end{array} ; \frac{(-1)^{p-q}}{z}\right),
\end{aligned}
$$

which is nothing but the formula (2.3). The proof of Theorem 2.1 is complete.

Second proof of Theorem 2.1. By using the statement (ii) in Lemma 3.1, we shall give another proof of Theorem 2.1. Let $g(\xi)$ be the formal $1 /(q-p)$ Borel transform of our formal series $\hat{f}(z)$

$$
\begin{aligned}
g(\xi) & =\left(\hat{\mathcal{B}}_{1 /(q-p)} \hat{f}\right)(\xi)=\sum_{n=0}^{\infty} \frac{(\boldsymbol{\alpha})_{n}}{(\boldsymbol{\gamma})_{n}\{(q-p) n\} !} \frac{\xi^{n}}{n !} \\
& =\sum_{n=0}^{\infty} \frac{(\boldsymbol{\alpha})_{n}}{(\boldsymbol{\gamma})_{n}((\boldsymbol{q}-\boldsymbol{p}) /(q-p))_{n}} \frac{1}{n !}\left(\frac{\xi}{(q-p)^{q-p}}\right)^{n} \\
& ={ }_{q} F_{q-1}\left(\begin{array}{c}
\boldsymbol{\alpha} \\
\boldsymbol{\gamma},(\boldsymbol{q}-\boldsymbol{p}) /(q-p)
\end{array} ; \frac{\xi}{(q-p)^{q-p}}\right)
\end{aligned}
$$

where

$$
\frac{\boldsymbol{q}-\boldsymbol{p}}{q-p}=\left(\frac{1}{q-p}, \ldots, \frac{q-p-1}{q-p}, 1\right), \quad\left(\frac{\boldsymbol{q}-\boldsymbol{p}}{q-p}\right)_{n}=\prod_{k=1}^{q-p}\left(\frac{k}{q-p}\right)_{n}
$$


Then we can see that $g(\xi)$ has the similar properties with $h(\xi)$ in the previous proof. Therefore, the Borel sum $f(z)$ is given by the following Laplace integral

$$
\begin{aligned}
f(z) & =\left(\mathcal{L}_{1 /(q-p)} g\right)(z) \\
& =\frac{1}{z^{1 /(q-p)}} \int_{0}^{\infty(d)} \exp \left[-\left(\frac{\xi}{z}\right)^{1 /(q-p)}\right] g(\xi) d\left(\xi^{1 /(q-p)}\right),
\end{aligned}
$$

where $d \neq 0(\bmod 2 \pi),|d-\arg z|<(q-p) \pi / 2$ and $\arg \xi=d$. We can regard that $f(z)$ is analytic in $S(\pi,(q-p+2) \pi, \infty)$ by rotating the argument of the path of the integration in (3.16). By a change of variable, we have

$$
f(z)=\int_{0}^{\infty(b)} e^{-u} g\left(z u^{q-p}\right) d u
$$

where $b=(d-\arg z) /(q-p)$. We employ the Barnes integral representation of $g(\xi)$ which is given by

$$
g(\xi)=\frac{C_{1}}{2 \pi i} \int_{I} \frac{\Gamma(\boldsymbol{\alpha}+\zeta) \Gamma(-\zeta)}{\Gamma(\boldsymbol{\gamma}+\zeta) \Gamma((\boldsymbol{q}-\boldsymbol{p}) /(q-p)+\zeta)}\left(-\frac{\xi}{(q-p)^{(q-p)}}\right)^{\zeta} d \zeta
$$

where $|\arg (-\xi)|<\pi$,

$$
C_{1}=C_{\alpha \gamma} \times \Gamma\left(\frac{\boldsymbol{q}-\boldsymbol{p}}{q-p}\right)
$$

and the path of integration $I$ is the same one as in (3.10). Moreover by taking residues on the left side of $I$ in the expression (3.18), we can exchange the order of integrations in (3.17) and we have

$$
f(z)=\frac{C_{1}}{2 \pi i} \int_{I} \frac{\Gamma(\boldsymbol{\alpha}+\zeta) \Gamma(-\zeta)(-z)^{\zeta}}{\Gamma(\boldsymbol{\gamma}+\zeta) \Gamma((\boldsymbol{q}-\boldsymbol{p}) /(q-p)+\zeta)} \frac{\Gamma(1+(q-p) \zeta)}{(q-p)^{(q-p) \zeta}} d \zeta
$$

Now by using the following multiplication formula (cf. [Erd 2, p.4]) with $m=$ $2,3, \ldots$ 


$$
\begin{aligned}
& \prod_{k=0}^{m-1} \Gamma\left(\zeta+\frac{k}{m}\right)=(2 \pi)^{(m-1) / 2} m^{\frac{1}{2}-m \zeta} \Gamma(m \zeta), \\
& \zeta+\frac{k}{m} \notin \mathbb{Z}_{\leq 0}(k=0,1, \ldots, m-1),
\end{aligned}
$$

we have

$$
\frac{\Gamma(1+(q-p) \zeta)}{\Gamma((\boldsymbol{q}-\boldsymbol{p}) /(q-p)+\zeta)}=(2 \pi)^{-(q-p-1) / 2} \cdot(q-p)^{\frac{1}{2}+(q-p) \zeta}
$$

and

$$
\Gamma\left(\frac{\boldsymbol{q}-\boldsymbol{p}}{q-p}\right)=(2 \pi)^{(q-p-1) / 2}(q-p)^{-\frac{1}{2}} .
$$

Therefore we obtain

$$
f(z)=\frac{C_{\alpha \gamma}}{2 \pi i} \int_{I} \frac{\Gamma(\boldsymbol{\alpha}+\zeta) \Gamma(-\zeta)}{\Gamma(\gamma+\zeta)}(-z)^{\zeta} d \zeta
$$

This is nothing but the formula (3.11) in the previous proof.

Proof of Theorem 2.2. From (3.11), we get the following formula for the difference between the Borel sum and one which is obtained after rotating around the origin.

$$
\begin{aligned}
f(z)-f\left(z e^{2 \pi i}\right) & =\frac{C_{\alpha \gamma}}{2 \pi i} \int_{I} \frac{\Gamma(\boldsymbol{\alpha}+\zeta) \Gamma(-\zeta)}{\Gamma(\boldsymbol{\gamma}+\zeta)}(-z)^{\zeta}\left(1-e^{2 \pi i \zeta}\right) d \zeta \\
& =\frac{C_{\alpha \gamma}}{2 \pi i} \int_{I} \frac{\Gamma(\boldsymbol{\alpha}+\zeta) \Gamma(-\zeta)}{\Gamma(\boldsymbol{\gamma}+\zeta)} z^{\zeta}(-2 i) \sin (\pi \zeta) d \zeta
\end{aligned}
$$

By using the reciprocal formula (3.14) to the term $-\Gamma(-\zeta) \sin (\pi \zeta)$, this expression becomes

$$
f(z)-f\left(z e^{2 \pi i}\right)=C_{\alpha \gamma} \int_{I} \frac{\Gamma(\boldsymbol{\alpha}+\zeta)}{\Gamma(\boldsymbol{\gamma}+\zeta) \Gamma(1+\zeta)} z^{\zeta} d \zeta
$$

Finally by the residue theorem, we get the desired formula (2.5).

\section{§4. Proof of Theorem 2.3}

Proof of Theorem 2.3. First, it should be remarked that the Borel sum $f(z)$ is given by the following formula (3.11)

$$
f(z)=\frac{C_{\alpha \gamma}}{2 \pi i} \int_{I} \frac{\Gamma(\boldsymbol{\alpha}+\zeta) \Gamma(-\zeta)}{\Gamma(\boldsymbol{\gamma}+\zeta)}(-z)^{\zeta} d \zeta, \quad z \in S(\pi,(q-p+2) \pi, \infty)
$$


Theorefore what we have to do is to calculate carefully the residues of the integrand which has a simple pole at each point $\zeta=-\alpha_{j}-n(3 \leq j \leq q, n=$ $0,1, \ldots)$ and $\zeta=-\alpha_{1}-k(0 \leq k \leq m-1)$ (and no simple pole at $\zeta=-\alpha_{1}-k$ if $m=0)$, and has a double pole at each point $\zeta=-\alpha_{2}-n(n=0,1, \ldots)$.

By denoting by $\operatorname{Res}(c)$ the residue at $\zeta=c$ of the integrand in (4.1), we have

$$
\begin{aligned}
& =C_{\alpha \gamma}\left\{\sum_{k=0}^{m-1} \operatorname{Res}\left(-\alpha_{1}-k\right)+\sum_{n=0}^{\infty} \operatorname{Res}\left(-\alpha_{2}-n\right)+\sum_{j=3}^{q} \sum_{n=0}^{\infty} \operatorname{Res}\left(-\alpha_{j}-n\right)\right\} \\
& \overline{\overline{\text { put }}} C_{\alpha \gamma}\left\{I_{1}+I_{2}+I_{3}\right\} .
\end{aligned}
$$

Here we denote each sum in $(4.2)$ by $I_{j}(j=1,2,3)$ in order. The term $I_{3}$ is the same one as in the proof of Theorem 2.1 and we have

$$
I_{3}=\sum_{j=3}^{q} C_{\alpha \gamma}(j)(-z)^{-\alpha_{j}} F_{q-1}\left(\begin{array}{c}
\alpha_{j}, 1+\alpha_{j}-\gamma \\
1+\alpha_{j}-\widehat{\boldsymbol{\alpha}_{j}}
\end{array} ; \frac{(-1)^{p-q}}{z}\right) .
$$

Next, in order to calculate $I_{1}$ and $I_{2}$, by using the formula $\Gamma(1+z)=z \Gamma(z)$, we deform $\Gamma\left(\alpha_{1}+\zeta\right) \Gamma\left(\alpha_{2}+\zeta\right)$, a part of the term of the integrand, to the form

$$
\Gamma\left(\alpha_{1}+\zeta\right) \Gamma\left(\alpha_{2}+\zeta\right)=\frac{\Gamma^{2}\left(\alpha_{2}+\zeta\right)}{\left(\alpha_{1}+\zeta\right)\left(\alpha_{1}+\zeta+1\right) \cdots\left(\alpha_{1}+\zeta+m-1\right)} .
$$

Then we can see that

$$
\begin{aligned}
I_{1}= & \sum_{k=0}^{m-1}\left(\zeta+\alpha_{1}+k\right) \frac{\Gamma^{2}\left(\alpha_{2}+\zeta\right)}{\left(\alpha_{1}+\zeta\right)\left(\alpha_{1}+\zeta+1\right) \cdots\left(\alpha_{1}+\zeta+m-1\right)} \\
& \times\left.\frac{\prod_{j=3}^{q} \Gamma\left(\alpha_{j}+\zeta\right) \Gamma(-\zeta)}{\Gamma(\gamma+\zeta)}(-z)^{\zeta}\right|_{\zeta=-\alpha_{1}-k} ^{q} \\
= & \sum_{k=0}^{m-1} \frac{(-1)^{k}}{k !} \frac{\Gamma^{2}(m-k)}{(m-k-1) !} \times \frac{\prod_{j=3}^{q} \Gamma\left(\alpha_{j}-\alpha_{1}-k\right) \Gamma\left(\alpha_{1}+k\right)}{\Gamma\left(\gamma-\alpha_{1}-k\right)}(-z)^{-\alpha_{1}-k}
\end{aligned}
$$

By using the formula (3.13) and recalling $m=\alpha_{2}-\alpha_{1}$, we obtain

$$
I_{1}=C_{\alpha \gamma}(1)(-z)^{-\alpha_{1}} \sum_{k=0}^{m-1} \frac{\left(\alpha_{1}\right)_{k}\left(1+\alpha_{1}-\gamma\right)_{k}}{\left(1+\alpha_{1}-\widehat{\boldsymbol{\alpha}_{1}}\right)_{k}} \frac{1}{k !}\left(\frac{(-1)^{p-q}}{z}\right)^{k} .
$$


For $I_{2}$ we have to calculate the residues carefully as follows, because the order of pole is 2 .

$$
\begin{aligned}
& I_{2}=\sum_{n=0}^{\infty} \frac{d}{d \zeta}\left(\zeta+\alpha_{2}+n\right)^{2} \frac{\Gamma^{2}\left(\alpha_{2}+\zeta\right)}{\left(\alpha_{1}+\zeta\right)\left(\alpha_{1}+\zeta+1\right) \cdots\left(\alpha_{1}+\zeta+m-1\right)} \\
& \times\left.\frac{\prod_{j=3}^{q} \Gamma\left(\alpha_{j}+\zeta\right) \Gamma(-\zeta)}{\Gamma(\gamma+\zeta)}(-z)^{\zeta}\right|_{\zeta=-\alpha_{2}-n} \\
& =\sum_{n=0}^{\infty}\left\{\left.\frac{d}{d \zeta}\left(\zeta+\alpha_{2}+n\right)^{2} \Gamma^{2}\left(\alpha_{2}+\zeta\right)\right|_{\zeta=-\alpha_{2}-n}\right. \\
& \times \frac{1}{\left(\alpha_{1}-\alpha_{2}-n\right) \cdots\left(\alpha_{1}-\alpha_{2}-n+m-1\right)} \\
& \times \frac{\prod_{j=3}^{q} \Gamma\left(\alpha_{j}-\alpha_{2}-n\right) \Gamma\left(\alpha_{2}+n\right)}{\Gamma\left(\gamma-\alpha_{2}-n\right)}(-z)^{-\alpha_{2}-n} \\
& +\left.\left(\zeta+\alpha_{2}+n\right)^{2} \Gamma^{2}\left(\alpha_{2}+\zeta\right)\right|_{\zeta=-\alpha_{2}-n} \\
& \times\left.\frac{d}{d \zeta} \frac{1}{\left(\alpha_{1}+\zeta\right) \cdots\left(\alpha_{1}+\zeta+m-1\right)}\right|_{\zeta=-\alpha_{2}-n} \\
& \times \frac{\prod_{j=3}^{q} \Gamma\left(\alpha_{j}-\alpha_{2}-n\right) \Gamma\left(\alpha_{2}+n\right)}{\Gamma\left(\gamma-\alpha_{2}-n\right)}(-z)^{-\alpha_{2}-n} \\
& +\left.\left(\zeta+\alpha_{2}+n\right)^{2} \Gamma^{2}\left(\alpha_{2}+\zeta\right)\right|_{\zeta=-\alpha_{2}-n} \\
& \times \frac{1}{\left(\alpha_{1}-\alpha_{2}-n\right) \cdots\left(\alpha_{1}-\alpha_{2}-n+m-1\right)} \\
& \times\left.\frac{d}{d \zeta} \frac{\prod_{j=3}^{q} \Gamma\left(\alpha_{j}+\zeta\right) \Gamma(-\zeta)}{\Gamma(\gamma+\zeta)}\right|_{\zeta=-\alpha_{2}-n}(-z)^{-\alpha_{2}-n} \\
& +\left.\left(\zeta+\alpha_{2}+n\right)^{2} \Gamma^{2}\left(\alpha_{2}+\zeta\right)\right|_{\zeta=-\alpha_{2}-n} \\
& \times \frac{1}{\left(\alpha_{1}-\alpha_{2}-n\right) \cdots\left(\alpha_{1}-\alpha_{2}-n+m-1\right)} \\
& \left.\times\left.\frac{\prod_{j=3}^{q} \Gamma\left(\alpha_{j}-\alpha_{2}-n\right) \Gamma\left(\alpha_{2}+n\right)}{\Gamma\left(\gamma-\alpha_{2}-n\right)} \frac{d}{d \zeta}(-z)^{\zeta}\right|_{\zeta=-\alpha_{2}-n}\right\} \\
& \overline{\overline{\mathrm{put}}} \sum_{n=0}^{\infty}\left\{I_{21}(n)+I_{22}(n)+I_{23}(n)+I_{24}(n)\right\} \text {. }
\end{aligned}
$$

Here we denote each sum in (4.6) by $I_{2 j}(n)(j=1,2,3,4)$ in order.

Now, we put

$$
A(\zeta)=\left(\zeta+\alpha_{2}+n\right) \Gamma\left(\alpha_{2}+\zeta\right)
$$


Then by using the formula $\Gamma(1+z)=z \Gamma(z)$, we have

$$
\begin{aligned}
A(\zeta) & =\left(\zeta+\alpha_{2}+n\right) \frac{\Gamma\left(\alpha_{2}+\zeta+n+1\right)}{\left(\alpha_{2}+\zeta\right)\left(\alpha_{2}+\zeta+1\right) \cdots\left(\alpha_{2}+\zeta+n\right)} \\
& =\frac{\Gamma\left(\alpha_{2}+\zeta+n+1\right)}{\left(\alpha_{2}+\zeta\right)\left(\alpha_{2}+\zeta+1\right) \cdots\left(\alpha_{2}+\zeta+n-1\right)}
\end{aligned}
$$

which implies

$$
A\left(-\alpha_{2}-n\right)=\frac{(-1)^{n}}{n !} .
$$

Moreover, by taking logarithmic derivative of both sides of the expression (4.7) we get

$$
\frac{A^{\prime}(\zeta)}{A(\zeta)}=\Psi\left(\alpha_{2}+\zeta+n+1\right)-\frac{1}{\alpha_{2}+\zeta}-\cdots-\frac{1}{\alpha_{2}+\zeta+n-1},
$$

where $\Psi(z)=\Gamma^{\prime}(z) / \Gamma(z)$ denotes the Psi function. This shows

$$
\begin{aligned}
A^{\prime}\left(-\alpha_{2}-n\right) & =A\left(-\alpha_{2}-n\right)\left(\Psi(1)+\frac{1}{n}+\frac{1}{n-1}+\cdots+\frac{1}{1}\right) \\
& =\frac{(-1)^{n}}{n !} \Psi(1+n),
\end{aligned}
$$

where we have used some formula for the Psi function given in [Erd 2, p.16]. Then from (4.8) and (4.10) we obtain

$$
\begin{aligned}
I_{21}(n)= & 2 \frac{1}{(n !)^{2}} \Psi(1+n) \frac{(-1)^{m} n !}{(m+n) !} \\
& \times \frac{\prod_{j=3}^{q} \Gamma\left(\alpha_{j}-\alpha_{2}-n\right) \Gamma\left(\alpha_{2}+n\right)}{\Gamma\left(\gamma-\alpha_{2}-n\right)}(-z)^{-\alpha_{2}-n}, \\
I_{22}(n)= & \frac{1}{(n !)^{2}} \frac{(-1)^{m} n !}{(m+n) !}\left(\frac{1}{m+n}+\cdots+\frac{1}{n+1}\right) \\
& \times \frac{\prod_{j=3}^{q} \Gamma\left(\alpha_{j}-\alpha_{2}-n\right) \Gamma\left(\alpha_{2}+n\right)}{\Gamma\left(\gamma-\alpha_{2}-n\right)}(-z)^{-\alpha_{2}-n}, \\
I_{24}(n)= & \frac{1}{(n !)^{2}} \frac{(-1)^{m} n !}{(m+n) !} \\
& \times \frac{\prod_{j=3}^{q} \Gamma\left(\alpha_{j}-\alpha_{2}-n\right) \Gamma\left(\alpha_{2}+n\right)}{\Gamma\left(\gamma-\alpha_{2}-n\right)}(-z)^{-\alpha_{2}-n} \log (-z) .
\end{aligned}
$$

In order to calculate $I_{23}(n)$, we put

$$
B(\zeta)=\frac{\prod_{j=3}^{q} \Gamma\left(\alpha_{j}+\zeta\right) \Gamma(-\zeta)}{\Gamma(\gamma+\zeta)}
$$


By taking logarithmic derivative of both sides of (4.14), we get

$$
B^{\prime}(\zeta)=B(\zeta)\left(\sum_{j=3}^{q} \Psi\left(\alpha_{j}+\zeta\right)-\Psi(-\zeta)-\Psi(\gamma+\zeta)\right)
$$

This shows

$$
\begin{aligned}
\left.\frac{d}{d \zeta} B(\zeta)\right|_{\zeta=-\alpha_{2}-n}=\frac{\prod_{j=3}^{q} \Gamma\left(\alpha_{j}-\alpha_{2}-n\right) \Gamma\left(\alpha_{2}+n\right)}{\Gamma\left(\gamma-\alpha_{2}-n\right)} \\
\quad \times\left(\sum_{j=3}^{q} \Psi\left(\alpha_{j}-\alpha_{2}-n\right)-\Psi\left(\alpha_{2}+n\right)-\Psi\left(\gamma-\alpha_{2}-n\right)\right)
\end{aligned}
$$

From this, we have

$$
\begin{gathered}
I_{23}(n)=\frac{1}{(n !)^{2}} \frac{(-1)^{m} n !}{(m+n) !} \frac{\prod_{j=3}^{q} \Gamma\left(\alpha_{j}-\alpha_{2}-n\right) \Gamma\left(\alpha_{2}+n\right)}{\Gamma\left(\gamma-\alpha_{2}-n\right)} \\
\times\left(\sum_{j=3}^{q} \Psi\left(\alpha_{j}-\alpha_{2}-n\right)-\Psi\left(\alpha_{2}+n\right)-\Psi\left(\gamma-\alpha_{2}-n\right)\right)(-z)^{-\alpha_{2}-n} .
\end{gathered}
$$

Therefore, from (4.11), (4.12), (4.13) and (4.17), and by using the formula

$$
\Psi(1+n+m)=\Psi(1+n)+\frac{1}{n+1}+\frac{1}{n+2}+\cdots+\frac{1}{n+m},
$$

(cf. [Erd 2, p.16]), we obtain the following

$$
\begin{aligned}
I_{2}=\sum_{n=0}^{\infty} & \frac{(-1)^{m}}{n !(m+n) !} \frac{\prod_{j=3}^{q} \Gamma\left(\alpha_{j}-\alpha_{2}-n\right) \Gamma\left(\alpha_{2}+n\right)}{\Gamma\left(\gamma-\alpha_{2}-n\right)}(-z)^{-\alpha_{2}-n} \\
& \times\{\log (-z)+\Psi(1+n)+\Psi(1+n+m) \\
& \left.\quad+\sum_{j=3}^{q} \Psi\left(\alpha_{j}-\alpha_{2}-n\right)-\Psi\left(\alpha_{2}+n\right)-\Psi\left(\gamma-\alpha_{2}-n\right)\right\} .
\end{aligned}
$$

Finally, from (4.3), (4.5), (4.18) and the formula (3.13) we obtain the desired formula (2.6).

\section{Remark 4.}

(i) In the case where there are many pairs $\left(\alpha_{i}, \alpha_{j}\right)$ with an integer difference but there are no pair $\left(\alpha_{i}, \gamma_{j}\right)$ with an integer difference, a similar result is proved 
by a similar mannar. The difference in the result is the appearence of powers and products of the logarithmic term and higher derivatives of Psi function.

(ii) In Theorem 2.3 if we further add an assumption that $\gamma_{1}-\alpha_{1} \in \mathbb{Z}$, then more complicated situations occur which are shown in the below.

Let $\gamma_{1}-\alpha_{1}=\ell \in \mathbb{Z}$. We should remark that the Borel sum is always given by the formula (4.1). What we have to do is to calculate the residues $I_{j}$ $(j=1,2)$ where $I_{3}$ in $(4.2)$ is the same one as in (4.3).

(I) The case $\ell \leq 0$. In this case, by using the formula $\Gamma(1+z)=z \Gamma(z)$ we deform a part of the terms of the integrand to the form

$$
\frac{\Gamma\left(\alpha_{1}+\zeta\right) \Gamma\left(\alpha_{2}+\zeta\right)}{\Gamma\left(\gamma_{1}+\zeta\right)}=\left(\gamma_{1}+\zeta\right)\left(\gamma_{1}+\zeta+1\right) \cdots\left(\gamma_{1}-\ell-1+\zeta\right) \Gamma\left(\alpha_{2}+\zeta\right) .
$$

This shows that the integrand has a simple pole at each point $\zeta=-\alpha_{j}-n$ $(2 \leq j \leq q, n=0,1, \ldots)$. This implies that $I_{1}=0$ in (4.2) because $-\alpha_{1}-k$ $(0 \leq k \leq m-1)$ are all regular points for the integrand. Next we have

$$
\begin{aligned}
I_{2}= & \sum_{n=0}^{\infty} \operatorname{Res}\left(-\alpha_{2}-n\right) \\
= & \sum_{n=0}^{\infty}\left(\gamma_{1}-\alpha_{2}-n\right) \cdots\left(\gamma_{1}-\ell-1-\alpha_{2}-n\right) \frac{(-1)^{n}}{n !} \\
& \quad \times \frac{\prod_{j=3}^{q} \Gamma\left(\alpha_{j}-\alpha_{2}-n\right) \Gamma\left(\alpha_{2}+n\right)}{\Gamma\left(\widehat{\gamma_{1}}-\alpha_{2}-n\right)}(-z)^{-\alpha_{2}-n} \\
= & \sum_{n=0}^{\infty}(-1)^{\ell+n} \frac{(m+n-\ell) !}{n !(m+n) !} \frac{\prod_{j=3}^{q} \Gamma\left(\alpha_{j}-\alpha_{2}-n\right) \Gamma\left(\alpha_{2}+n\right)}{\Gamma\left(\widehat{\gamma_{1}}-\alpha_{2}-n\right)}(-z)^{-\alpha_{2}-n} \\
= & \frac{(-1)^{\ell}(m-\ell) !}{m !} C_{\alpha \gamma}\left(2 ; \widehat{\alpha_{1}}, \widehat{\gamma_{1}}\right)(-z)^{-\alpha_{2}}{ }_{p} F_{q-1}\left(\begin{array}{c}
\alpha_{2}, 1+\alpha_{2}-\gamma \\
1+\alpha_{2}-\widehat{\alpha_{2}}
\end{array} \frac{(-1)^{p-q}}{z}\right),
\end{aligned}
$$

where

$$
C_{\alpha \gamma}\left(2 ; \widehat{\alpha_{1}}, \widehat{\gamma_{1}}\right)=\frac{\Gamma\left(\alpha_{2}\right) \prod_{\ell=1, \ell \neq 1,2}^{q} \Gamma\left(\alpha_{\ell}-\alpha_{2}\right)}{\Gamma\left(\widehat{\gamma_{1}}-\alpha_{2}\right)} .
$$

Therefore, from (4.3) and (4.19) we obtain the following

$$
\begin{aligned}
& \text { 4.20) } f(z)=C_{\alpha \gamma}\left[\sum_{j=3}^{q} C_{\alpha \gamma}(j)(-z)^{-\alpha_{j}}{ }_{p} F_{q-1}\left(\begin{array}{c}
\alpha_{j}, 1+\alpha_{j}-\gamma \\
1+\alpha_{j}-\widehat{\boldsymbol{\alpha}_{j}}
\end{array} \frac{(-1)^{p-q}}{z}\right)\right. \\
& \left.+\frac{(-1)^{\ell}(m-\ell) !}{m !} C_{\alpha \gamma}\left(2 ; \widehat{\alpha_{1}}, \widehat{\gamma_{1}}\right)(-z)^{-\alpha_{2}}{ }_{p} F_{q-1}\left(\begin{array}{c}
\alpha_{2}, 1+\alpha_{2}-\gamma \\
1+\alpha_{2}-\widehat{\boldsymbol{\alpha}_{2}}
\end{array} ; \frac{(-1)^{p-q}}{z}\right)\right] .
\end{aligned}
$$


(II) The case $0<\ell \leq m$. From

$$
\frac{\Gamma\left(\alpha_{1}+\zeta\right) \Gamma\left(\alpha_{2}+\zeta\right)}{\Gamma\left(\gamma_{1}+\zeta\right)}=\frac{\Gamma\left(\alpha_{2}+\zeta\right)}{\left(\alpha_{1}+\zeta\right)\left(\alpha_{1}+\zeta+1\right) \cdots\left(\alpha_{1}+\ell-1+\zeta\right)},
$$

we see that the integrand has a simple pole at each point $\zeta=-\alpha_{1}-k(0 \leq$ $k \leq \ell-1)$ and $\zeta=-\alpha_{j}-n(2 \leq j \leq q, n=0,1, \ldots)$. This implies that $I_{1}$ is given by (4.5) (but in the summation $k$ runs from 0 to $\ell-1$ ) and $I_{2}$ is the same one as (4.19).

(III) The case $\ell>m$. From

$$
\begin{aligned}
& \frac{\Gamma\left(\alpha_{1}+\zeta\right) \Gamma\left(\alpha_{2}+\zeta\right)}{\Gamma\left(\gamma_{1}+\zeta\right)} \\
& \quad=\frac{1}{\left(\alpha_{1}+\zeta\right) \cdots\left(\alpha_{1}+m-1+\zeta\right)} \frac{\Gamma\left(\alpha_{2}+\zeta\right)}{\left(\alpha_{2}+\zeta\right) \cdots\left(\alpha_{2}+\ell-m-1+\zeta\right)},
\end{aligned}
$$

we see that the integrand has a simple pole at each point $\zeta=-\alpha_{1}-k(0 \leq$ $k \leq m-1), \zeta=-\alpha_{2}-i(i \geq \ell-m)$ and $\zeta=-\alpha_{j}-n(3 \leq j \leq q, n=0,1, \cdots)$, and a double pole at each point $\zeta=-\alpha_{2}-i(0 \leq i \leq \ell-m-1)$. This implies that $I_{1}$ is given by $(4.5)$ and

$$
I_{2}=\left\{\sum_{i=0}^{\ell-m-1}+\sum_{i=\ell-m}^{\infty}\right\} \operatorname{Res}\left(-\alpha_{2}-i\right) .
$$

By taking into considerations (4.18) and (4.19), we obtain

$$
\begin{aligned}
& I_{2}=\sum_{i=0}^{\ell-m-1} \frac{(-1)^{m}}{i !(m+i) !} \frac{\prod_{j=3}^{q} \Gamma\left(\alpha_{j}-\alpha_{2}-i\right) \Gamma\left(\alpha_{2}+i\right)}{\Gamma\left(\gamma-\alpha_{2}-i\right)}(-z)^{-\alpha_{2}-i} \\
& \times\{\log (-z)+\Psi(1+i)+\Psi(1+i+m) \\
& \left.\quad+\sum_{j=3}^{q} \Psi\left(\alpha_{j}-\alpha_{2}-i\right)-\Psi\left(\alpha_{2}+i\right)-\Psi\left(\gamma-\alpha_{2}-i\right)\right\} \\
& +\sum_{i=\ell-m}^{\infty} \frac{(-1)^{\ell+i}(m+i-\ell) !}{i !(m+i) !} \frac{\prod_{j=3}^{q} \Gamma\left(\alpha_{j}-\alpha_{2}-i\right) \Gamma\left(\alpha_{2}+i\right)}{\Gamma\left(\widehat{\gamma_{1}}-\alpha_{2}-i\right)}(-z)^{-\alpha_{2}-i}
\end{aligned}
$$

Summing up the above observations, we obtain the following

Corollary 4.1. Let us assume $\alpha_{2}-\alpha_{1}=m(m \geq 0), \alpha_{j}-\alpha_{1} \notin \mathbb{Z}$ $(3 \leq j \leq q), \gamma_{1}-\alpha_{1}=\ell \in \mathbb{Z}$ and $\gamma_{k}-\alpha_{1} \notin \mathbb{Z}(2 \leq k \leq p-1)$. Then the Borel sum $f(z)$ is given as follows. 
(i) The case $\ell \leq m$.

$$
\begin{aligned}
& \text {.22) } \frac{f(z)}{C_{\alpha \gamma}}=C_{\alpha \gamma}(1)(-z)^{-\alpha_{1}} \sum_{k=0}^{\ell-1} \frac{\left(\alpha_{1}\right)_{k}\left(1+\alpha_{1}-\gamma\right)_{k}}{\left(1+\alpha_{1}-\widehat{\boldsymbol{\alpha}_{1}}\right)_{k}} \frac{1}{k !}\left(\frac{(-1)^{p-q}}{z}\right)^{k} \\
& +\sum_{j=3}^{q} C_{\alpha \gamma}(j)(-z)^{-\alpha_{j}} F_{q-1}\left(\begin{array}{l}
\alpha_{j}, 1+\alpha_{j}-\gamma \\
1+\alpha_{j}-\widehat{\boldsymbol{\alpha}_{j}}
\end{array} \frac{(-1)^{p-q}}{z}\right) \\
& +\frac{(-1)^{\ell}(m-\ell) !}{m !} C_{\alpha \gamma}\left(2 ; \widehat{\alpha_{1}}, \widehat{\gamma_{1}}\right)(-z)^{-\alpha_{2}}{ }_{p} F_{q-1}\left(\begin{array}{c}
\alpha_{2}, 1+\alpha_{2}-\gamma \\
1+\alpha_{2}-\widehat{\boldsymbol{\alpha}_{2}}
\end{array} ; \frac{(-1)^{p-q}}{z}\right),
\end{aligned}
$$

where

$$
C_{\alpha \gamma}\left(2 ; \widehat{\alpha_{1}}, \widehat{\gamma_{1}}\right)=\frac{\Gamma\left(\alpha_{2}\right) \prod_{\ell=1, \ell \neq 1,2}^{q} \Gamma\left(\alpha_{\ell}-\alpha_{2}\right)}{\Gamma\left(\widehat{\gamma_{1}}-\alpha_{2}\right)} .
$$

Here we use the abbreviation that $\sum_{k=0}^{\ell-1} \cdots=0$ if $\ell \leq 0$. Moreover in this case the logarithmic term disappears.

(ii) The case $\ell>m$.

$$
\begin{aligned}
& \frac{f(z)}{C_{\alpha \gamma}}=C_{\alpha \gamma}(1)(-z)^{-\alpha_{1}} \sum_{k=0}^{m-1} \frac{\left(\alpha_{1}\right)_{k}\left(1+\alpha_{1}-\gamma\right)_{k}}{\left(1+\alpha_{1}-\widehat{\boldsymbol{\alpha}_{1}}\right)_{k}} \frac{1}{k !}\left(\frac{(-1)^{p-q}}{z}\right)^{k} \\
+ & \sum_{j=3}^{q} C_{\alpha \gamma}(j)(-z)^{-\alpha_{j}} F_{q-1}\left(\begin{array}{l}
\alpha_{j}, 1+\alpha_{j}-\gamma \\
1+\alpha_{j}-\widehat{\boldsymbol{\alpha}_{j}}
\end{array} \frac{(-1)^{p-q}}{z}\right) \\
+ & \frac{(-1)^{m}}{m !} C_{\alpha \gamma}\left(2 ; \widehat{\alpha_{1}}\right)(-z)^{-\alpha_{2}} \sum_{i=0}^{\ell-m-1} \frac{\left(\alpha_{2}\right)_{i}\left(1+\alpha_{2}-\gamma\right)_{i}}{\left(1+\alpha_{2}-\widehat{\boldsymbol{\alpha}_{2}}\right)_{i}} \frac{1}{i !}\left(\frac{(-1)^{p-q}}{z}\right)^{i} \\
\times & \{\log (-z)+\Psi(1+i)+\Psi(1+i+m) \\
+ & \left.\sum_{j=3}^{q} \Psi\left(\alpha_{j}-\alpha_{2}-i\right)-\Psi\left(\alpha_{2}+i\right)-\Psi\left(\gamma-\alpha_{2}-i\right)\right\} \\
+ & \frac{(-1)^{\ell}}{m !} C_{\alpha \gamma}\left(2 ; \widehat{\alpha_{1}}, \widehat{\gamma_{1}}\right)(-z)^{-\alpha_{2}} \\
\times & \sum_{i=\ell-m}^{\infty} \frac{(m-\ell+i) !\left(\alpha_{2}\right)_{i}\left(1+\alpha_{2}-\widehat{\gamma_{1}}\right)_{i}}{\left(1+\alpha_{2}-\widehat{\alpha_{2}}\right)_{i}}\left(\frac{(-1)^{p-q}}{z}\right)^{i}
\end{aligned}
$$

where $C_{\alpha \gamma}\left(2 ; \widehat{\alpha_{1}}\right)$ is the same one as in Theorem 2.3 . 


\section{$\S 5 . \quad$ Application to a Partial Differential Equation}

As a mention in Section 1, we shall give an application to a partial differential equation of Theorem 2.2. Let us consider the Cauchy problem which is given in (1.2)

$$
\left\{\begin{array}{l}
\partial_{t}^{p} u(t, x)=\partial_{x}^{q} u(t, x), \\
u(0, x)=\varphi(x), \quad \partial_{t}^{j} u(0, x)=0(1 \leq j \leq p-1) .
\end{array}\right.
$$

This Cauchy problem has a unique formal solution

$$
\hat{u}(t, x)=\sum_{n=0}^{\infty} \frac{\varphi^{(q n)}(x)}{(p n) !} t^{p n} .
$$

By the assumption that $q>p$, this formal power series is divergent in general. Miyake [Miy] proved the following theorem for the characterization of the Borel summability of the formal solution (5.2).

Theorem B (Miyake). Let $\hat{u}(t, x)$ be the formal solution (5.2) of the Cauchy problem (5.1). Then $\hat{u}(t, x)$ is $p /(q-p)$-summable in d direction in $t$-plane if and only if the following two conditions for the Cauchy data $\varphi(x)$ are satisfied:

(i) $\varphi(x)$ can be continued analytically in $q$ sectors

$$
\Omega(p, q ; d, \varepsilon)=\bigcup_{j=0}^{q-1} S\left(\frac{p d+2 \pi j}{q}, \varepsilon, \infty\right)
$$

for some $\varepsilon>0$.

(ii) $\varphi(x)$ has the growth condition of exponential order at most $q /(q-p)$, that is, there exist positive constants $C$ and $\delta$ such that we have

$$
|\varphi(x)| \leq C \exp \left(\delta|x|^{q /(q-p)}\right), \quad x \in \Omega(p, q ; d, \varepsilon) .
$$

Under the above conditions, we get the integral representation of the Borel sum by using the kernel function as follows, which is a generalization of a result in Lutz-Miyake-Schäfke [LMS].

Theorem 5.1 (Integral representation of Borel sum). Under the conditions for the Cauchy data $\varphi(x)$ in Theorem B, the Borel sum $u(t, x)$ of the formal solution (5.2) of the Cauchy problem (5.1) is given by

$$
u(t, x)=\int_{0}^{\infty(p d / q)} \Phi(x, \zeta) k(t, \zeta) d \zeta
$$


where

$$
\Phi(x, \zeta)=\sum_{j=0}^{q-1} \varphi\left(x+\zeta \omega^{j}\right), \quad \omega=\exp (2 \pi i / q),
$$

and the kernel function $k(t, \zeta)$ is given by

$$
\begin{aligned}
k(t, \zeta)=\frac{1}{\zeta} & \times \frac{\Gamma(\boldsymbol{p} / p)}{\Gamma(\boldsymbol{q} / q)} \times \sum_{j=1}^{q-1} \frac{\Gamma\left(\widehat{(\boldsymbol{q} / q)_{j}}-j / q\right)}{\Gamma(\boldsymbol{p} / p-j / q)}\left(\frac{q^{q}}{p^{p}} \frac{t^{p}}{\zeta^{q}}\right)^{-j / q} \\
& \times{ }_{p} F_{q-1}\left(\begin{array}{l}
1+j / q-\boldsymbol{p} / p \\
1+j / q-\widehat{(\boldsymbol{q} / q)_{j}}
\end{array} ;(-1)^{p-q} \frac{p^{p}}{q^{q}} \frac{\zeta^{q}}{t^{p}}\right)
\end{aligned}
$$

where $\boldsymbol{p}=(1,2, \ldots, p)$ and $\boldsymbol{q}=(1,2, \ldots, q)$.

Proof of Theorem 5.1. Let $v(s, x)$ be the $(q-p)$ times iterated $p$-Borel transforms in $t$-variable of the formal solution $\hat{u}(t, x)$

$$
v(s, x)=\left(\left(\hat{\mathcal{B}}_{p}\right)^{q-p} \hat{u}\right)(s, x)=\sum_{n=0}^{\infty} \frac{\varphi^{(q n)}(x)}{(p n) !} \frac{s^{p n}}{(n !)^{q-p}} .
$$

By Cauchy's integral formula, for sufficiently small $|s|$ and $|x|$ we have

$$
\begin{aligned}
v(s, x) & =\frac{1}{2 \pi i} \oint_{|\zeta|=r} \frac{\varphi(x+\zeta)}{\zeta} \sum_{n=0}^{\infty} \frac{(q n) !}{(p n) !(n !)^{q-p}}\left(\frac{s^{p}}{\zeta^{q}}\right)^{n} d \zeta \\
& =\frac{1}{2 \pi i} \oint_{|\zeta|=r} \frac{\varphi(x+\zeta)}{\zeta} h(s, \zeta) d \zeta,
\end{aligned}
$$

where $r>\sqrt[q]{\left(q^{q} / p^{p}\right)\left|s^{p}\right|}$ and $h(s, \zeta)={ }_{q} F_{q-1}\left(\boldsymbol{q} / q ; \boldsymbol{p} / p, 1, \ldots, 1 ; q^{q} s^{p} / p^{p} \zeta^{q}\right)$. Here we notice that $h(s, \zeta)$ has $q$ singular points in $\zeta$-plane at $q$ roots of $\zeta^{q}=$ $\left(q^{q} / p^{p}\right) s^{p}$, which we denote by $\zeta=\sqrt[q]{\left(q^{q} / p^{p}\right) s^{p}}$ for short, for a fixed $s \neq 0$ with $\arg s=d$. We put $a=\left(q^{q} / p^{p}\right)^{1 / q} s^{p / q}$ (the root with argument $d p / q$ ), and we denote by $[0, a]$ the segment joining the origin and $a$. Then $h(s, \zeta)$ is univalent in $\mathbb{C}_{\zeta} \backslash \bigcup_{j=0}^{q-1}\left[0, a \omega^{j}\right]$ (outside of $q$ segments). Therefore by deforming the contour of integration (5.9) on these $q$ segments, we get the following expression for $v(s, x)$

$$
v(s, x)=\frac{1}{2 \pi i} \int_{0}^{a} \frac{\Phi(x, \zeta)}{\zeta}\left\{h(s, \zeta)-h\left(s, \zeta \omega^{-1}\right)\right\} d \zeta .
$$

Here we remark in the expression (5.10) that the integrand is integrable at $\zeta=0$ and $\zeta=a$. In fact, we notice that $w(z)=h(s, \zeta)$ with $z=q^{q} s^{p} / p^{p} \zeta^{q}$ satisfies 
$E_{q, q-1}[\boldsymbol{q} / q ; \boldsymbol{p} / p, 1, \ldots, 1](w)=0$. The characteristic exponents at $z=1$ are $\{0,1,2, \ldots, q-2, \nu\}$ where

$$
\nu=\sum_{\ell=1}^{p} \frac{\ell}{p}+(q-p-1)-\sum_{m=1}^{q} \frac{m}{q}(>-1),
$$

which implies the integrability at $\zeta=a$. Next, the characteristic exponents at $z=\infty$ are $\{1 / q, 2 / q, \ldots, 1\}$, which implies the integrability at $\zeta=0$.

Since $h(s, \zeta)$ is univalent outside of $q$ segments, we have

$$
v(s, x)=\frac{1}{2 \pi i} \int_{0}^{\infty(p d / q)} \frac{\Phi(x, \zeta)}{\zeta}\left\{h(s, \zeta)-h\left(s, \zeta \omega^{-1}\right)\right\} d \zeta .
$$

Hence the Borel sum $u(t, x)$ is given by the following iterated Laplace transforms of $v$

$$
\text { (5.13) } \begin{aligned}
u(t, x) & =\left(\left(\mathcal{L}_{p}\right)^{q-p} v\right)(t, x) \\
& =\frac{1}{2 \pi i} \int_{0}^{\infty(p d / q)} \frac{\Phi(x, \zeta)}{\zeta}\left\{\left(\mathcal{L}_{p}\right)^{q-p}\left(h(\cdot, \zeta)-h\left(\cdot, \zeta \omega^{-1}\right)\right)\right\}(t, \zeta) d \zeta .
\end{aligned}
$$

This observation shows that the kernel function $k(t, \zeta)$ is given by

$$
k(t, \zeta)=\frac{1}{2 \pi i} \frac{1}{\zeta}\left\{\left(\left(\mathcal{L}_{p}\right)^{q-p} h\right)(\cdot, \zeta)-\left(\left(\mathcal{L}_{p}\right)^{q-p} h\right)\left(\cdot, \zeta \omega^{-1}\right)\right\}(t, \zeta) .
$$

Now, we shall prove that the function $h(s, \zeta) / \zeta$ is an iterated formal Borel transforms of the formal solution of the following Cauchy problem for the adjoint equation

$$
\left\{\begin{array}{l}
\partial_{t}^{p} u(t, \zeta)=\left(-\partial_{\zeta}\right)^{q} u(t, \zeta) \\
u(0, \zeta)=1 / \zeta, \quad \partial_{t}^{j} u(0, \zeta)=0(1 \leq j \leq p-1)
\end{array}\right.
$$

This Cauchy problem (5.15) has a unique formal solution

$$
\begin{aligned}
\hat{e}(t, \zeta) & =\frac{1}{\zeta} \sum_{n=0}^{\infty} \frac{(q n) !}{(p n) !}\left(\frac{t^{p}}{\zeta^{q}}\right)^{n} \\
& =\frac{1}{\zeta}{ }_{q} F_{p-1}\left(\frac{\boldsymbol{q}}{q} ; \frac{\widehat{\boldsymbol{p}_{p}}}{p} ; \frac{q^{q}}{p^{p}} \frac{t^{p}}{\zeta^{q}}\right) \underset{\mathrm{put}}{\bar{\zeta}} \frac{1}{\zeta} \hat{f}(z),
\end{aligned}
$$

where $z=q^{q} / p^{p} \times t^{p} / \zeta^{q}$ and $\hat{f} \in \mathbb{C}[[z]]_{q-p}$. Let $g(\xi)=\left(\left(\hat{\mathcal{B}}_{1}\right)^{q-p} \hat{f}\right)(\xi)$. Then we can see that

$$
g\left(\frac{q^{q}}{p^{p}} \frac{s^{p}}{\zeta^{q}}\right)=h(s, \zeta)
$$


Hence, if we put $f(z)$ be the Borel sum of $\hat{f}(z)$, then $f(z)=\left(\left(\mathcal{L}_{1}\right)^{q-p} g\right)(z)$ and the Borel sum $e(t, \zeta)$ of $\hat{e}(t, \zeta)$ is given by

$$
e(t, \zeta)=\frac{1}{\zeta} f(z)
$$

Thus we can see that the kernel function $k(t, \zeta)$ is given by

$$
\begin{aligned}
k(t, \zeta) & =\frac{1}{2 \pi i}\left\{e(t, \zeta)-e\left(t e^{\frac{2 \pi i}{p}}, \zeta\right)\right\} \\
& =\frac{1}{2 \pi i} \times \frac{1}{\zeta}\left\{f(z)-f\left(z e^{2 \pi i}\right)\right\}, \quad z=q^{q} t^{p} / p^{p} \zeta^{q} .
\end{aligned}
$$

Therefore by using Theorem 2.2, we get the kernel function which is given by (5.7). This completes the proof of Theorem 5.1.

\section{$\S 6 . \quad$ Explicit Form of the Kernel in Special Cases}

We shall prove the explicit representation of the kernels stated in Thoerem 5.1 in the following special cases:

\begin{tabular}{|c|c|}
\hline$(p, q)$ & $k(t, \zeta)$ \\
\hline$(1,2)$ & $\frac{1}{\sqrt{4 \pi t}} e^{-\zeta^{2} / 4 t} \quad$ (heat kernel) \\
\hline$(2,3)$ & $\sqrt{\frac{3}{4 \pi}} \frac{1}{\zeta} \exp \left(-\frac{1}{2} \times \frac{4 \zeta^{3}}{27 t^{2}}\right) W_{1 / 2,1 / 6}\left(\frac{4 \zeta^{3}}{27 t^{2}}\right)$ \\
\hline$(1,3)$ & $\frac{1}{(3 t)^{1 / 3}} A i\left(\frac{\zeta}{(3 t)^{1 / 3}}\right)$ \\
\hline
\end{tabular}

In the above table $W_{a, b}$ denotes the Whittaker function (cf. [Erd 2]) and $A i$ denotes the Airy function (cf. [Erd 1]) which is given by

$$
\begin{aligned}
A i(z) & =\frac{1}{\pi} \int_{0}^{\infty} \cos \left(\frac{t^{3}}{3}+z t\right) d t, \\
A i(-z) & =\frac{z^{1 / 2}}{3}\left(J_{1 / 3}(\xi)+J_{-1 / 3}(\xi)\right), \quad \xi=\frac{2}{3} z^{3 / 2},
\end{aligned}
$$

where $J_{c}$ denotes the Bessel function of the order $c$ which has the following power series expansion

$$
J_{c}(z)=\sum_{n=0}^{\infty} \frac{(-1)^{n}}{n ! \Gamma(n+c+1)}\left(\frac{z}{2}\right)^{2 n+c} .
$$


The case $(p, q)=(1,2)$. We omit the proof (cf. [LMS]).

The case $(p, q)=(1,3)$. From Theorem 5.1 we obtain

$$
\begin{aligned}
k(t, \zeta)=\frac{1}{\zeta} & \frac{1}{\Gamma(1 / 3) \Gamma(2 / 3)} \times\left\{\frac{\Gamma(2 / 3-1 / 3) \Gamma(3 / 3-1 / 3)}{\Gamma(1 / 1-1 / 3)}\left(3^{3} \frac{t}{\zeta^{3}}\right)^{-1 / 3}\right. \\
& \times{ }_{1} F_{2}\left(\begin{array}{c}
1+1 / 3-1 / 1 \\
1+1 / 3-2 / 3,1+1 / 3-3 / 3
\end{array} ;(-1)^{1-3} \frac{\zeta^{3}}{3^{3} t}\right) \\
+ & \frac{\Gamma(1 / 3-2 / 3) \Gamma(3 / 3-2 / 3)}{\Gamma(1 / 1-2 / 3)}\left(3^{3} \frac{t}{\zeta^{3}}\right)^{-2 / 3} \\
& \left.\times{ }_{1} F_{2}\left(1+2 / 3-1 / 3,1+2 / 3-3 / 3 ;(-1)^{1-3} \frac{\zeta^{3}}{3^{3} t}\right)\right\} \\
= & \frac{1}{\zeta} \frac{1}{\Gamma(1 / 3) \Gamma(2 / 3)}\left\{\Gamma(1 / 3)\left(\frac{27 t}{\zeta^{3}}\right)^{-1 / 3}{ }_{0} F_{1}\left({ }^{2} \frac{2}{3} ;(-1)^{-2} \frac{\zeta^{3}}{27 t}\right)\right. \\
+ & \left.\Gamma(-1 / 3)\left(\frac{27 t}{\zeta^{3}}\right)^{-2 / 3}{ }_{0} F_{1}\left({ }^{3} ;{ }^{3} ;(-1)^{-2} \frac{\zeta^{3}}{27 t}\right)\right\}
\end{aligned}
$$

We notice the following relation

$$
{ }_{0} F_{1}(\quad ; 1+c ; z)=\Gamma(1+c)\left(\frac{z}{-1}\right)^{-c / 2} J_{c}\left(2\left(\frac{z}{-1}\right)^{1 / 2}\right) \quad c \in \mathbb{C}
$$

where and in what follows we use the abbreviation that $-1=e^{\pi i}$ and therefore $z /(-1)=e^{-\pi i} z, z /(-1)^{3}=e^{-3 \pi i} z$, etc. Then by using this relation, we get

${ }_{0} F_{1}\left(; \frac{2}{3} ;(-1)^{-2} \frac{\zeta^{3}}{27 t}\right)=\Gamma(2 / 3)\left(\frac{\zeta^{3}}{(-1)^{3} 27 t}\right)^{1 / 6} J_{-1 / 3}\left(2\left(\frac{\zeta^{3}}{(-1)^{3} 27 t}\right)^{1 / 2}\right)$,

$$
{ }_{0} F_{1}\left(; \frac{4}{3} ;(-1)^{-2} \frac{\zeta^{3}}{27 t}\right)=\Gamma(4 / 3)\left(\frac{\zeta^{3}}{(-1)^{3} 27 t}\right)^{-1 / 6} J_{1 / 3}\left(2\left(\frac{\zeta^{3}}{(-1)^{3} 27 t}\right)^{1 / 2}\right)
$$


By substituting these formulas to the expression (6.4), we have

(6.8) $k(t, \zeta)=\frac{1}{\zeta} \frac{1}{\Gamma(1 / 3) \Gamma(2 / 3)}$

$$
\begin{aligned}
& \times\left\{\Gamma(1 / 3) \Gamma(2 / 3)\left(\frac{\zeta^{3}}{(-1) 27 t}\right)^{1 / 2} J_{-1 / 3}\left(2\left(\frac{\zeta^{3}}{(-1)^{3} 27 t}\right)^{1 / 2}\right)\right. \\
& \left.\quad+\Gamma(-1 / 3) \Gamma(4 / 3)\left(\frac{(-1) \zeta^{3}}{27 t}\right)^{1 / 2} J_{1 / 3}\left(2\left(\frac{\zeta^{3}}{(-1)^{3} 27 t}\right)^{1 / 2}\right)\right\}
\end{aligned}
$$

We notice the formula $\Gamma(-1 / 3) \Gamma(4 / 3)=\Gamma(1 / 3) \Gamma(2 / 3) /(-1)$ which is obtained from the fundamental relation $\Gamma(1+z)=z \Gamma(z)$ and we obtain

$$
k(t, \zeta)=\left(\frac{\zeta}{(-1) 27 t}\right)^{1 / 2}\left\{J_{-1 / 3}\left(2 \sqrt{\frac{\zeta^{3}}{(-1)^{3} 27 t}}\right)+J_{1 / 3}\left(2 \sqrt{\frac{\zeta^{3}}{(-1)^{3} 27 t}}\right)\right\} .
$$

Therefore, the formula (6.2) implies the desired formula

$$
k(t, \zeta)=\frac{1}{(3 t)^{1 / 3}} A i\left(\frac{\zeta}{(3 t)^{1 / 3}}\right) .
$$

The case $(p, q)=(2,3)$. From Theorem 5.1 we obtain

$$
\begin{aligned}
k(t, \zeta)= & \frac{\Gamma(1 / 2)}{\Gamma(1 / 3) \Gamma(2 / 3)} \times \frac{1}{\zeta}\left\{\frac{\Gamma(2 / 3-1 / 3) \Gamma(3 / 3-1 / 3)}{\Gamma(1 / 2-1 / 3) \Gamma(2 / 2-1 / 3)}\left(\frac{27 t^{2}}{4 \zeta^{3}}\right)^{-1 / 3}\right. \\
& \times{ }_{2} F_{2}\left(\begin{array}{l}
1+1 / 3-1 / 2,1+1 / 3-2 / 2 \\
1+1 / 3-2 / 3,1+1 / 3-3 / 3
\end{array}(-1)^{2-3} \frac{4 \zeta^{3}}{27 t^{2}}\right) \\
+ & \frac{\Gamma(1 / 3-2 / 3) \Gamma(3 / 3-2 / 3)}{\Gamma(1 / 2-2 / 3) \Gamma(2 / 2-2 / 3)}\left(\frac{27 t^{2}}{4 \zeta^{3}}\right)^{-2 / 3} \\
& \left.\times{ }_{2} F_{2}\left(\begin{array}{l}
1+2 / 3-1 / 2,1+2 / 3-2 / 2 \\
1+2 / 3-1 / 3,1+2 / 3-3 / 3
\end{array}(-1)^{2-3} \frac{4 \zeta^{3}}{27 t^{2}}\right)\right\} \\
= & \frac{\Gamma(1 / 2)}{\Gamma(1 / 3) \Gamma(2 / 3)} \frac{1}{\zeta}\left\{\frac{\Gamma(1 / 3)}{\Gamma(1 / 6)}\left(\frac{4 \zeta^{3}}{27 t^{2}}\right)^{1 / 3}{ }_{1} F_{1}\left(\frac{5}{6} ; \frac{2}{3} ;(-1)^{-1} \frac{4 \zeta^{3}}{27 t^{2}}\right)\right. \\
+ & \left.\frac{\Gamma(-1 / 3)}{\Gamma(-1 / 6)}\left(\frac{4 \zeta^{3}}{27 t^{2}}\right)^{2 / 3}{ }_{1} F_{1}\left(\frac{7}{6} ; \frac{4}{3} ;(-1)^{-1} \frac{4 \zeta^{3}}{27 t^{2}}\right)\right\}
\end{aligned}
$$


We recall the following two formulas:

In case of $2 m \notin \mathbb{Z}$,

$$
W_{k, m}(z)=\frac{\Gamma(-2 m)}{\Gamma(1 / 2-m-k)} M_{k, m}(z)+\frac{\Gamma(2 m)}{\Gamma(1 / 2+m-k)} M_{k,-m}(z),
$$

where

$$
M_{k, m}(z)=\exp \left(-\frac{z}{2}\right) z^{1 / 2+m}{ }_{1} F_{1}(1 / 2+m-k ; 1+2 m ; z), \quad|z|<\infty,
$$

(cf. [Tem, p.178]).

For any $\alpha, \gamma \in \mathbb{C}\left(\gamma \notin \mathbb{Z}_{\leq 0}\right)$,

$$
{ }_{1} F_{1}(\alpha ; \gamma ; z)=e^{z}{ }_{1} F_{1}(\gamma-\alpha ; \gamma ;-z)
$$

Then by using the formula (6.13), the expression (6.10) becomes

$$
\begin{aligned}
& k(t, \zeta)=\frac{\Gamma(1 / 2)}{\Gamma(1 / 3) \Gamma(2 / 3)} \frac{1}{\zeta} \exp \left(-\frac{4 \zeta^{3}}{27 t^{2}}\right) \\
& \quad \times\left\{\frac{\Gamma(1 / 3)}{\Gamma(1 / 6)}\left(\frac{4 \zeta^{3}}{27 t^{2}}\right)^{1 / 3}{ }_{1} F_{1}\left(-\frac{1}{6} ; \frac{2}{3} ; \frac{4 \zeta^{3}}{27 t^{2}}\right)\right. \\
&\left.\quad+\frac{\Gamma(-1 / 3)}{\Gamma(-1 / 6)}\left(\frac{4 \zeta^{3}}{27 t^{2}}\right)^{2 / 3}{ }_{1} F_{1}\left(\frac{1}{6} ; \frac{4}{3} ; \frac{4 \zeta^{3}}{27 t^{2}}\right)\right\},
\end{aligned}
$$

and finally by using the formulas (6.11) and (6.12) with $k=1 / 2, m=1 / 6$, we obtain

$$
k(t, \zeta)=\frac{\Gamma(1 / 2)}{\Gamma(1 / 3) \Gamma(2 / 3)} \frac{1}{\zeta} \exp \left(-\frac{1}{2} \times \frac{4 \zeta^{3}}{27 t^{2}}\right) W_{1 / 2,1 / 6}\left(\frac{4 \zeta^{3}}{27 t^{2}}\right) .
$$

\section{References}

[Bal 1] Balser, W., From Divergent Power Series to Analytic Functions, Springer Lecture Notes, No. 1582, 1994.

[Bal 2] — Divergent solutions of the heat equation: on an article of Lutz, Miyake and Schäfke, Pac. J. Math., 188 (1999), 53-63.

[BM] Balser, W. and Miyake, M., Summability of formal solutions of certain partial differential equations, Acta. Sci. Math. (Szeged), 65 (1999), 543-551.

[Bar] Barnes, E. W., The asymptotic expansion of integral functions defined by generalized hypergeometric series, Proc. London Math. Soc., (2) 5 (1907), 59-116.

[Bra] Braaksma, B. L. J., Multisummability of formal power series solutions of nonlinear meromorphic differential equations, Ann. Inst. Fourier Grenoble, 42 (1992), 517-540.

[Erd 1] Erdélyi, A., Asymptotic Expansion, Dover 1956. 
[Erd 2] Erdélyi, A., Magnus, W., Oberhettinger, F. and Tricomi, F. G., Higher Transcendental Functions, Vol. I. McGraw-Hill Book Company, INC. 1953.

[IKSY] Iwasaki, K., Kimura, H., Shimomura, S. and Yoshida, M., From Gauss to Painlevé, A Modern Theory of Special Functions, Aspects Math., 1991.

[Luk] Luke, Y. L., Integrals of Bessel Functions, McGraw-Hill Book Company, INC. 1962.

[LMS] Lutz, D., Miyake, M. and Schäfke, R., On the Borel summability of divergent solutions of the heat equation, Nagoya Math. J., 154 (1999), 1-29.

[Mei] Meijer, C. S., On the G-function. I, Indag. Math., 8 (1946) 124-134.

[Miy] Miyake, M., Borel Summability of Divergent Solutions of the Cauchy Problem to Non-Kowalevskian Equations, Proc. of Conf. on Partial Differential Equations and Their Appl. (edited by H. Chen and L. Rodino), World Scientific 1999, 225-239.

[MI] Miyake, M. and Ichinobe, K., On the Borel summability of divergent solutions of parabolic type equations and Barnes generalized hypergeometric functions, to appear in RIMS Kôkyûroku, Kyoto Univ., (2000).

[Ram] Ramis, J.-P., Les séries $k$-sommable et leurs applications, Springer Lecture Notes in Phys., 126 (1980), 178-199.

[Tem] Temme, Nico M., Special Functions, An Introduction to the Classical Functions of Mathematical Physics, John Wiley \& Sons, Inc. 1996. 